\title{
Increased prevalence of pelvic venous congestion sign on sacroiliac MRI in women with clinically suspected sacroiliitis
}

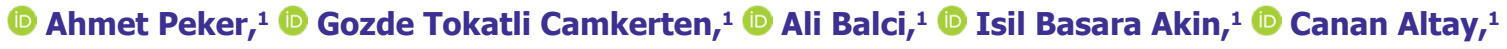 \\ (1) Gercek Can $^{2}$ \\ ${ }^{1}$ Department of Radiology, Dokuz Eylul University Faculty of Medicine, Izmir, Turkey \\ ${ }^{2}$ Department of Rheumatology and Immunology, Dokuz Eylul University Faculty of Medicine, Izmir, Turkey
}

\begin{abstract}
OBJECTIVE: To compare the prevalence of pelvic venous congestion (PVC) sign on sacroiliac and hip MRI in women of reproductive age as a possible cause of pain mimicking sacroiliitis.

METHODS: This retrospective study included 727 MRI examinations (401 sacroiliac joint MRI and 326 hip joint MRI) that were performed between January 2010 and December 2017. Images were evaluated for the presence of sacroilitis, presence of PVC sign and presence of other incidental findings of musculoskeletal and genitourinary disorders. After removing patients with other musculoskeletal and genitourinary disorders that may cause pain $(n=188)$, remaining 539 (322 sacroiliac and 217 hip), MRI examinations were re-analyzed for the presence of PVC sign.

RESULTS: Four hundred one patients with sacroiliac MRI examination had 120 (29.9\%) PVC sign and 326 patients with hip MRI examinations had $54(16.6 \%)$ PVC sign $(p<0.001)$. After removing patients with other musculoskeletal and genitourinary disorders that may cause pain, 322 patients with sacroiliac MRI had 102 (31.7\%) PVC sign and 217 patients with hip MRI examinations had $38(17.5 \%)$ PVC sign $(p<0.001)$. No significant difference was found between patients with acute sacroiliitis and patients without acute sacroiliitis concerning PVC prevalence $(p>0.05)$. There were also no significant differences between other comparable incidental findings.
\end{abstract}

CONCLUSION: Significantly increased PVC prevalence in sacroiliac MRI exams may be attributable to PCS simulating clinical sacroiliitis.

Keywords: Pelvic congestion syndrome; pelvic venous congestion sign; sacroiliitis.

Cite this article as: Peker A, Tokatli Camkerten G, Balci A, Basara Akin I, Altay C, Can G. Increased prevalence of pelvic venous congestion sign on sacroiliac MRI in women with clinically suspected sacroiliitis. North Clin Istanb 2020;7(6):551-556.

$\mathrm{P}$ elvic congestion syndrome (PCS) is a frequent condition that may cause chronic pelvic pain in women of reproductive age [1]. PCS was first described by Richet in 1857 as a chronic dull pelvic pain that arose from an increased venous pressure, and the severity of the illness is associated with the number and tortuosity of dilated veins [2]. Although different diagnostic crite- ria were defined in the recent studies like the presence of an ovarian vein diameter larger than $8 \mathrm{~mm}$ and/or parauterine vein diameter larger than $5 \mathrm{~mm}$ and vein flow velocity as less than $3 \mathrm{~cm} / \mathrm{s}$ or the presence of venous reflux, there is still no consensus for imaging diagnosis. Since the presence of dilated veins can also be seen in $10 \%$ of the normal population and the clinical 
findings do not always correlate with imaging, PCS diagnosis is established clinically. Magnetic resonance imaging (MRI) defined pelvic venous congestion (PVC) sign is an important finding of PCS [3-8].

Spondyloarthropathies $(\mathrm{SpA})$ are a group of joint diseases that include reactive arthritis, chronic inflammatory bowel disease with arthropathy, psoriatic arthritis, and undifferentiated spondyloarthropathy [9]. They are seronegative for rheumatoid factor; and they are often associated with the pres $\neg$ ence of human lymphocyte antigen (HLA)-B27 [10]. The sacroiliac joints are involved in most cases of axial SpA, with sacroiliitis usually being the first manifestation $[11,12]$. The time from the onset of symptoms to the time of diagnosis of $\mathrm{SpA}$ has been shown as five to 11 years in different studies. Sacroiliac MRI imaging is requested more and more by clinicians because of the increased treatment options and effectiveness at early-onset diagnosis in $\mathrm{SpA}[13,14]$. Presence of bone marrow edema (BME) on MRI is critical for diagnosing active sacroiliitis in the Assessment of SpondyloArthritis International Society (ASAS) classification [15].

Musculoskeletal disorders, such as hip joint diseases and pelvic disorders, may cause back pain in addition to SpA and PCS. The present study aims to compare the prevalence of PVC sign on sacroiliac and hip MRI and to evaluate other incidental findings that may cause back pain in women of reproductive age.

\section{MATERIALS AND METHODS}

This retrospective study was approved by the Instutional Ethics Committee on 19 July 2018 by issue number 2018/18-07. The requirement for informed consent was waived owing to the retrospective nature of this study.

\section{Study Population}

A retrospective search of our hospital was queried to identify patients who underwent sacroiliac joint MRI and hip joint MRI examinations between January 2010 and December 2017. Among these, we selected the female patients who were at the ages between 18 and 50 and who were referred from the departments of rheumatology, physiotherapy and rehabilitation, and orthopedics with the complaint of back pain. Patients who had active malignancy (23), patients who were screened for bone metastasis (27), patients who underwent MRI due to trauma (89) and patients who had a previous pelvic operation (72) were excluded from this study. Finally, 727 MRI examinations (401 sacroiliac joint MRI and 326 hip joint MRI) were included in this study. The mean age of the study population was $37.35 \pm 8.38$ years (range: 18-50).

\section{Imaging Protocol}

MRI examinations of the patients were performed using two different $1.5 \mathrm{~T}$ MRI units (Intera, software version 8.1; Philips Medical Systems, Eindhoven, The Netherlands -Gyroscan Achieva, Philips, ACS-NT, Bothell, WA, USA). Sacroiliac joint MRI sequence protocol included: para-coronal (along long the axis of the sacral bone perpendicular to the S2 vertebral body) T1-weighted turbo spin-echo (TSE) (slice thickness (ST):3 mm; repetition time/echo time (TR/TE):500/14 ms field of view $(F O V): 250 \mathrm{~mm} \times 250 \mathrm{~mm}$ ); para-coronal STIR (ST: 3 mm; TR/TE/TI: 2500/8/150 ms, FOV: $250 \mathrm{~mm} \times 250 \mathrm{~mm}$ ), and para-axial fat-saturated dual SPIR related to the pelvis (ST: $4.5 \mathrm{~mm}$; TR/TE1/TE2: 2000/100/13 ms; FOV: $280 \mathrm{~mm} \times 280 \mathrm{~mm}$ ) from L5 to the lesser trochanter. No cartilage enhancing gradient echo MRI sequences were obtained. As per ASAS guidelines, no contrast-enhanced pulse sequences were obtained [15].

Hip joint MRI examinations were bilateral. Hip joint MRI sequence protocol included: Coronal T1 weighted turbo spin-echo (TSE) (ST: $4 \mathrm{~mm}$; TR/TE: 540/7 ms, FOV: $420 \mathrm{~mm} \times 420 \mathrm{~mm}$ ); coronal dual echo SPIR (ST: $4 \mathrm{~mm}$; TR/TE1/TE2: 2137/100/8 ms, FOV: $400 \mathrm{~mm}$ $\times 400 \mathrm{~mm}$ ) and sagittal dual echo SPIR (ST: $3 \mathrm{~mm}$; TR/ TE1/TE2:1673/100/9/2 ms, FOV: $220 \mathrm{~mm} \times 220 \mathrm{~mm}$ ).

\section{Image Interpretation}

The MR images were reviewed in consensus for the presence of sacroiliitis, the presence of PVC sign and incidental findings by a fifth-year radiology resident, a fourthyear radiology resident and a musculoskeletal radiologist with 10 years of experience. The reviewers were blinded to clinical data and other imaging findings of the patients.

Sacroiliitis was diagnosed according to the previously defined ASAS criteria: BME is depicted as high signal on STIR images, typically located periarticularly. BME is highly suggestive of sacroiliitis when clearly present and located in the typical anatomical areas (sub-chondral or periarticular bone marrow). If there is only one signal (lesion) per MRI slice suggesting active inflammation, the lesion should be present on at least two consecutive 

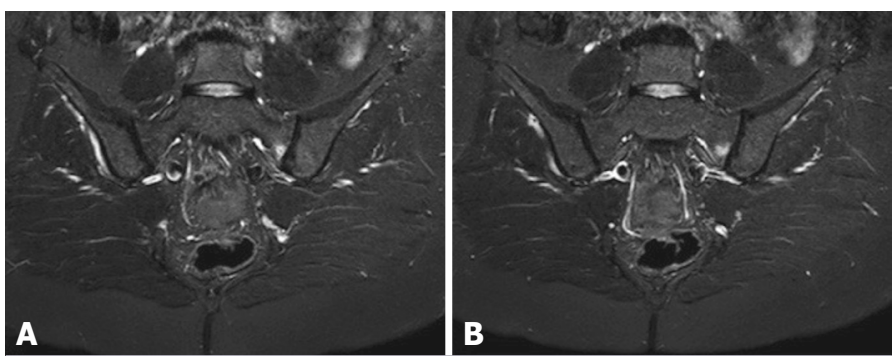

FIGURE 1. (A, B) Sacroiliac MRI of a 38-year-old patient with back pain. Para-coronal STIR images show peri-articular high signal intensity on two consecutive slices suggesting active sacroiliitis according to the ASAS criteria.

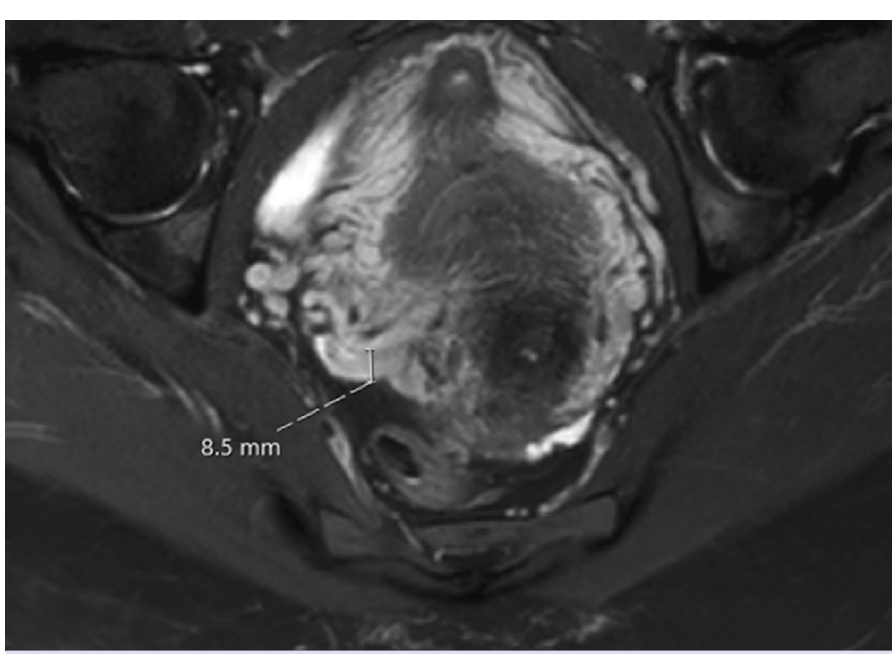

FIGURE2. Sacroiliac MRI of a 43-year-old female patient with back pain. Proton density para- axial image show bilaterally enlarged and tortuous veins of varying caliber, the largest measuring $8.5 \mathrm{~mm}$ on the right side.

slices. If there is more than one signal (lesion) on a single slice, one slice may be sufficient [15] (Fig. 1). PVC was considered if the patient had an ovarian vein diameter greater than $8 \mathrm{~mm}$ or had four or more ipsilateral parauterine veins with at least one of the veins diameter measured more than $4 \mathrm{~mm}[5,6,8]$ (Fig. 2, 3).

Incidental findings of musculoskeletal and genitourinary disorders were also recorded as present or absent:

- ovarian cysts larger than $30 \mathrm{~mm}$,

- uterine fibroids larger than $30 \mathrm{~mm}$,

- bladder wall trabeculation,

- endometriomas showing high signal intensity on $\mathrm{T} 1$ weighted and T2 weighted images or shading on T2W images,

- pelvic fluid with a depth of more than $20 \mathrm{~mm}$,

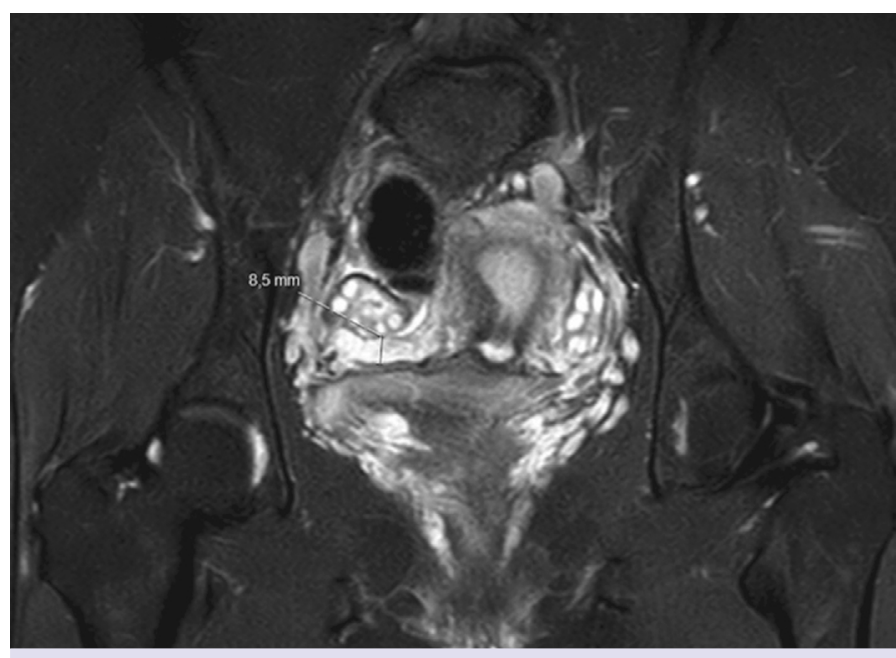

FIGURE 3. Hip joint MRI of a 34-year-old patient with hip pain. Coronal FS T2-weighted image shows dilated right ovarian vein measuring $8.5 \mathrm{~mm}$.

- insufficiency fractures,

- transitional vertebra with/without bone marrow edema,

- Modic Type 1 endplate changes (low signal intensity on T1 weighted images and high signal intensity on T2 weighted images),

- arthritis, femoroacetabular impingement, avascular necrosis, tendinitis and tendon tears also noted.

\section{Statistical Analysis}

Statistical analysis was performed using commercially available software (IBM SPSS Statistics for Windows, Version 21.0, IBM Corp., Armonk, NY, USA). Data were presented as mean \pm standard deviation or median and range for continuous variables. Kolmogorov-Smirnov test was used to assess the normal distribution of the data. Frequencies were compared using the chi-square test or Fisher's exact test as appropriate. Continuous variables were compared using Student's t-test. The results were evaluated within a $95 \%$ confidence interval. Statistical significance was set at $\mathrm{p}<0.05$.

\section{RESULTS}

Age

In this study, 727 MRI examinations (401 sacroiliac joint MRI and 326 hip joint MRI) were evaluated. The mean age of patients with sacroiliac joint MRI and hip joint MRI were $37.65 \pm 8.24$ and $36.98 \pm 8.54$, respectively $(\mathrm{p}>0.05)$. 
TABLE 1. Image interpretation results of the all study population for the presence of sacroiliitis and other incidental findings of musculoskeletal and genitourinary disorders

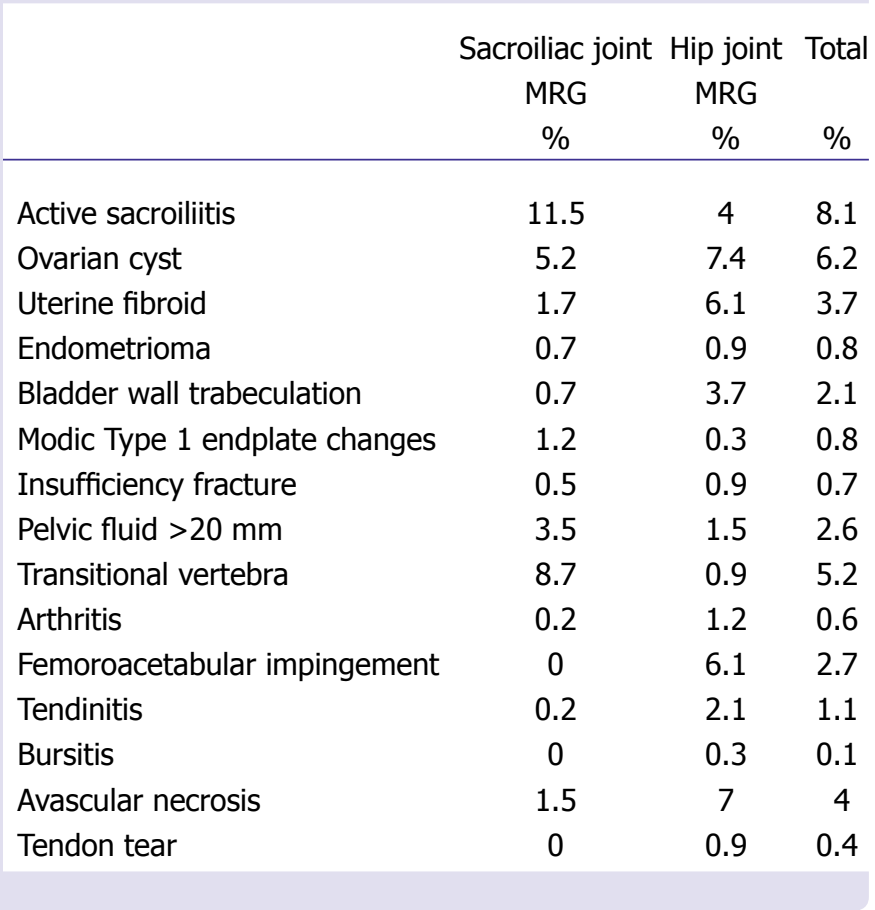

\section{Active Sacroiliitis and Incidental Findings of}

\section{Musculoskeletal and Genitourinary Disorders}

The presence of active sacroiliitis and other incidental findings of musculoskeletal and genitourinary disorders are displayed in Table 1. Active sacroiliitis was diagnosed in 46 (11.5\%) sacroiliac MRI and 13 (4\%) hip MRI resulting 59 (8.1\%) patients. Among incidental findings, the most frequent finding was ovarian cysts larger than $30 \mathrm{~mm}$ which was present in $45(6.2 \%) \mathrm{pa}-$ tients. Transitional vertebra anomaly was found in 38 $(5.2 \%)$ patients, and two of them had bone marrow edema. PVC was detected in 120 (29.9\%) patients who underwent sacroiliac joint MRI and in 54 (16.6\%) patients who underwent hip joint MRI (Table 2). There was a statistically significant difference between two groups $(\mathrm{p}<0.001)$.

After removing patients with the findings that may cause pain $(n=188$, shown in Table 1$)$, remaining 539 (322 sacroiliac and 217 hip) MRI examinations were analyzed separately for the presence of PVC. PVC was detected in $102(31.7 \%)$ patients who underwent sacroiliac joint MRI and in 38 (17.5\%) patients who underwent hip joint MRI (Table 3). There was still a statistically significant difference between two groups $(\mathrm{p}<0.001)$.
TABLE2. The prevalence of PVC demonstrated on sacroiliac joint MRI and hip joint MRI

\begin{tabular}{|c|c|c|c|c|}
\hline PVC & $\begin{array}{l}\text { Sacroiliac } \\
\text { joint MRG } \\
\quad \%\end{array}$ & $\begin{array}{l}\text { Hip } \\
\text { joint MRG } \\
\%\end{array}$ & $\%$ & $p$ \\
\hline Present & 29.9 & 16.6 & 23.9 & \\
\hline Absent & 70.1 & 83.4 & 76.1 & \\
\hline Total & 401 & 326 & 727 & $<0.001$ \\
\hline \multicolumn{5}{|c|}{$\begin{array}{l}\text { TABLE 3. The prevalence of PVC demonstrated on sacroiliac } \\
\text { joint MRI and hip joint MRI after removing patients with the } \\
\text { disorders that may cause pain }\end{array}$} \\
\hline \multirow[t]{2}{*}{ PVC } & $\begin{array}{c}\text { Sacroiliac } \\
\text { joint MRG }\end{array}$ & $\begin{array}{c}\text { Hip } \\
\text { joint MRG }\end{array}$ & Total & $p$ \\
\hline & $\%$ & & $\%$ & \\
\hline Present & 31.7 & 17.5 & 26 & \\
\hline Absent & 68.3 & 82.5 & 74 & \\
\hline Total & 322 & 217 & 539 & $<0.001$ \\
\hline
\end{tabular}

TABLE 4. Association of the PVC prevalence with active sacroiliitis

\begin{tabular}{lcccc} 
PVC & $\begin{array}{c}\text { Patients with } \\
\text { acute } \\
\text { sacroiliitis } \\
\%\end{array}$ & $\begin{array}{c}\text { Patients without } \\
\text { acute } \\
\text { sacroiliitis } \\
\%\end{array}$ & Total & $p$ \\
& 20.3 & 24.2 & $\%$ & \\
\hline Present & 79.7 & 75.8 & 76.1 & \\
Absent & 59 & 668 & 727 & 0.63 \\
Total & & & &
\end{tabular}

No significant difference was found between patients with acute sacroiliitis and patients without acute sacroiliitis in terms of PVC prevalence $(p>0.05)$ (Table 4). There were also no significant differences between other comparable incidental findings.

\section{DISCUSSION}

In our study, PVC was detected in $29.9 \%$ of the patients who underwent sacroiliac MRI and $16.6 \%$ in patients who underwent hip joint MRI. To our knowledge, there 
is only one study in the literature that evaluated PVC prevalence on sacroiliac MRI and it was reported as $5.3 \%$ [16]. According to the current literature, more than 15\% of women aged between 20-50 are diagnosed with pelvic varices, which are not always symptomatic [17] and the incidence of pelvic congestion syndrome in women aged between 18 and 50 has been estimated as 15\% [2], consistent with patients who underwent hip joint MRI in our study (16.6\%). To our knowledge, our study is the first study evaluating PVC on hip joint MRI. There was a significant difference between patients' sacroiliac joint MRI and hip joint MRI for the presence of PVC, even after we excluded patients with other findings that might cause pain. A significantly increased PVC prevalence in patients who underwent sacroiliac joint MRI may be attributed to that the PCS may simulate sacroiliitis clinically.

In $11.5 \%$ of the patients who underwent sacroiliac joint MRI with clinical suspicion of sacroiliitis were diagnosed with active sacroiliitis according to the recent ASAS criteria in our study. Besides, $54.9 \%$ of the patients who underwent sacroiliac joint MRI, there were no findings of musculoskeletal and genitourinary disorders, including PVC. This may reflect the unnecessary request for MRI in our country because MRI is cheap and easily accessible.

In our study, musculoskeletal findings that may cause pain was in $4.2 \%$ of patients who underwent sacroiliac joint MRI. Recent studies conducted by Jans et al. and Cimsit et al. $[16,18]$ reported the presence of non-inflammatory disease on sacroiliac joint MRI as $6 \%$. A lower frequency at our work may be because the findings we considered were different from their study. We considered only the findings that may cause back pain; however, they also considered the chronic-incidental findings that could be found in the normal population like disc degeneration and iliac wing cysts [19]. Lumbosacral transitional vertebra anomaly was seen in $5.2 \%$ of patients, which was reported as at least $4 \%$ of the population previously [20].

We found no significant differences between patients with acute sacroiliitis and patients without acute sacroiliitis concerning PVC prevalence. According to the study conducted by Cimsit et al. [16], a higher prevalence of PVC was reported in patients without MRI-defined sacroiliitis than patients with sacroiliitis. The reason for this may be due to the differences in criteria for diagnosing sacroiliitis. We considered active sacroiliitis, which may cause pain according to the recent ASAS criteria; they also considered fatty marrow deposition, erosions, sclerosis, ankylosis as well as bone marrow edema.

We had some limitations. Firstly, it was a retrospective study, and we did not perform any correlation with other imaging modalities. Secondly, patients' treatment and follow-up information were not available. Therefore, the exact number of patients who were diagnosed clinically as PCS was unknown. Thirdly, on which day of the menstrual cycle, the patients were at the time of MRI performed was not recorded. Fourthly, since MRI examinations were for visualization of sacroiliac joint and hip joint, entire genital organs, intervertebral discs and surrounding soft tissues were not completely included in the field of view. Lastly, we did not have a control group consisting of healthy individuals due to the respective nature of this study.

In conclusion, that a significantly increased PVC prevalence on sacroiliac MRI in women with clinically suspected sacroiliitis may be attributed to that PCS may simulate sacroiliitis clinically. Increasing the awareness of the PVC sign and keeping PVC in mind in the differential list of sacroiliitis may contribute to the correct diagnosis and treatment of these patients.

Ethics Committee Approval: This retrospective study was approved by the Dokuz Eylul University Non-Interventional Research Ethics Committee on 19 July 2018 by issue number 2018/18-07.

Conflict of Interest: No conflict of interest was declared by the authors.

Financial Disclosure: The authors declared that this study has received no financial support.

Authorship Contributions: Concept - AP, AB, GTC, IBA, GC, CA; Design - AP, AB, GTC, IBA, GC; Supervision - AP, AB, IBA, GC, CA; Materials - AP, AB, GTC, IBA, GC, CA; Data collection and/or processing - $A P, A B, G T C, I B A, G C, C A$; Analysis and/or interpretation - $A P$, $A B, G T C, I B A, G C, C A$; Literature review - AP, AB, GTC, IBA, GC, CA; Writing - $A P, A B, G T C$, IBA, GC, CA; Critical review - $A P, A B, G T C$, IBA, GC, CA.

\section{REFERENCES}

1. Koo S, Fan CM. Pelvic congestion syndrome and pelvic varicosities. Tech Vasc Interv Radiol 2014;17:90-5. [CrossRef]

2. Dorobisz TA, Garcarek JS, Kurcz J, Korta K, Dorobisz AT, Podgórski P, et al. Diagnosis and treatment of pelvic congestion syndrome: Singlecentre experiences. Adv Clin Exp Med 2017;26:269-76. [CrossRef]

3. Kuligowska E, Deeds L 3rd, Lu K 3rd. Pelvic pain: overlooked and underdiagnosed gynecologic conditions. Radiographics 2005;25:3-20.

4. Borghi C, Dell'Atti L. Pelvic congestion syndrome: the current state of the literature. Arch Gynecol Obstet 2016;293:291-301. [CrossRef]

5. Coakley FV, Varghese SL, Hricak H. CT and MRI of pelvic varices in women. J Comput Assist Tomogr 1999;23:429-34. [CrossRef] 
6. Knuttinen MG, Xie K, Jani A, Palumbo A, Carrillo T, Mar W. Pelvic venous insufficiency: imaging diagnosis, treatment approaches, and therapeutic issues. AJR Am J Roentgenol 2015;204:448-58. [CrossRef]

7. Tu FF, Hahn D, Steege JF. Pelvic congestion syndrome-associated pelvic pain: a systematic review of diagnosis and management. Obstet Gynecol Surv 2010;65:332-40. [CrossRef]

8. Maleux G, Stockx L, Wilms G, Marchal G. Ovarian vein embolization for the treatment of pelvic congestion syndrome: long-term technical and clinical results. J Vasc Interv Radiol 2000;11:859-64. [CrossRef]

9. Khan MA. Update on spondyloarthropathies. Ann Intern Med 2002;136:896-907. [CrossRef]

10. Braun J, Sieper J. Ankylosing spondylitis. Lancet 2007;369:1379-90.

11. Braun J, Sieper J. The sacroiliac joint in the spondyloarthropathies. Curr Opin Rheumatol 1996;8:275-87. [CrossRef]

12. Braun J, Bollow M, Sieper J. Radiologic diagnosis and pathology of the spondyloarthropathies. Rheum Dis Clin North Am 1998;24:697-735.

13. Guillemin F, Briançon S, Pourel J, Gaucher A. Long-term disability and prolonged sick leaves as outcome measurements in ankylosing spondylitis. Possible predictive factors. Arthritis Rheum 1990;33:1001-6.

14. Feldtkeller E, Khan MA, van der Heijde D, van der Linden S, Braun J. Age at disease onset and diagnosis delay in HLA-B27 negative vs. posi- tive patients with ankylosing spondylitis. Rheumatol Int 2003;23:61-6. 15. Sieper J, Rudwaleit M, Baraliakos X, Brandt J, Braun J, Burgos-Vargas $\mathrm{R}$, et al. The Assessment of SpondyloArthritis international Society (ASAS) handbook: a guide to assess spondyloarthritis. Ann Rheum Dis 2009;68 Suppl 2:ii1-44. [CrossRef]

16. Cimsit C, Yoldemir T, Tureli D, Aribal ME. Evaluation of sacroiliac joint MRI for pelvic venous congestion signs in women clinically suspected of sacroiliitis. Acta Radiol 2017;58:849-55. [CrossRef]

17. Wozniak S. Chronic pelvic pain. Ann Agric Environ Med 2016;23:2236. [CrossRef]

18. Jans L, Van Praet L, Elewaut D, Van den Bosch F, Carron P, Jaremko $\mathrm{JL}$, et al. MRI of the SI joints commonly shows non-inflammatory disease in patients clinically suspected of sacroiliitis. Eur J Radiol 2014;83:179-84. [CrossRef]

19. Cheung KM, Karppinen J, Chan D, Ho DW, Song YQ, Sham P, et al. Prevalence and pattern of lumbar magnetic resonance imaging changes in a population study of one thousand forty-three individuals. Spine (Phila Pa 1976) 2009;34:934-40. [CrossRef]

20. Konin GP, Walz DM. Lumbosacral transitional vertebrae: classification, imaging findings, and clinical relevance. AJNR Am J Neuroradiol 2010;31:1778-86. [CrossRef] 